\section{Implicit Event}

Jonas Mellin $^{1,2}$ and Mikael Berndtsson ${ }^{1,2}$

${ }^{1}$ University of Skövde, The Informatics

Research Centre, Skövde, Sweden

${ }^{2}$ University of Skövde, School of Informatics,

Skövde, Sweden

\section{Definition}

In active databases, an implicit event is an event that is implied by an ECA rule definition.

\section{Key Points}

ECA rules were developed as an optimization of condition action rules. The performance of rule evaluation was improved by allowing, or even requiring, explicit definition of when rules should be triggered in the form of events. However, it turns out that it ispossible to derive events from the condition in a meaningful way in some cases, and, thus, there are events that can be implied by the ECA rule definition. For example, if the condition is a logical expression $A=5 \wedge B=3$, then a disjunction of the events representing update to the variables can trigger the rule. That is, with implicit events (IF $A=5 \wedge B=3 \mathbf{D O}$ action) is equivalent to $\left(\mathbf{O N}\right.$ update $_{A}$ or update $_{B}$ IF $A=5$ $\wedge B=3$ DO action) with explicit events.

\section{Cross-References}

- Atomic Event

- Composite Event

$\checkmark$ ECA Rules

$>$ Event

- Event Detection

- Event Specification

$\checkmark$ Explicit Event 